\title{
Acute upper airway failure and mediastinal emphysema following a wire-guided percutaneous cricothyrotomy in a patient with severe maxillofacial trauma
}

\author{
R. Barkhuysen - M. A. W. Merkx - P. A. van Damme • \\ O. R. Buyne - F. J. A. van den Hoogen
}

Published online: 23 April 2008

(C) The Author(s) 2008

\begin{abstract}
Background In the presence of severe maxillofacial trauma, management of the airway is important because this condition poses a significant threat to airway patency. That securing the airway is not always straightforward is described and illustrated in this paper.

Case We present the case of a 23-year-old patient who sustained severe maxillofacial injury for which airway control was necessary. A wire-guided percutaneous dilation cricothyrotomy was performed, which was most probably the cause of an acute loss of airway patency. The literature regarding the role of percutaneous techniques in an elective and emergency setting is reviewed.
\end{abstract}

Keywords Percutaneous tracheotomy · Maxillofacial trauma $\cdot$ Emergency $\cdot$ Complications

\section{Introduction}

There are different techniques for creating a secure airway. Among these are the more or less noninvasive oral and

R. Barkhuysen · M. A. W. Merkx $(\varangle) \cdot$ P. A. van Damme

Department of Oral and Maxillofacial Surgery,

Radboud University Nijmegen Medical Centre,

P.O. Box 9101, Nijmegen, HB 6500, The Netherlands

e-mail: M.Merkx@mka.umcn.nl

O. R. Buyne

General Surgery, Radboud University Nijmegen Medical Centre,

Nijmegen, The Netherlands

F. J. A. van den Hoogen

Otorhinolaryngology,

Radboud University Nijmegen Medical Centre,

Nijmegen, The Netherlands nasal endotracheal intubations, whereas cricothyrotomy, conventional open tracheostomy (COT), and percutaneous cricothyrotomy and tracheotomy (PCT) provide a more aggressive means to this purpose. Indications for tracheotomy in maxillofacial trauma patients include all situations with obstruction or impending obstruction of the upper airway (Table 1) [19]. In the presence of severe maxillofacial trauma with gross displacement of bones and extensive soft tissue lacerations, a safe and definitive airway is crucial because this condition poses a significant threat to airway patency, and deterioration of the airway may occur rapidly. That securing the airway is not always straightforward is described and illustrated in the following case.

\section{Case report}

A 23-year-old elevator technician was transferred to the emergency ward after sustaining severe maxillofacial trauma when the elevator he was repairing accidentally went up, thereby squeezing his face between the top of the elevator and the roof of the elevator shaft.

Upon presentation, we saw a fully alert, cooperative patient, in a characteristic prone position with his neck somewhat anteflexed to maintain a patent airway. Endotracheal intubation at the scene was not attempted because of severe mutilation of the oral cavity (Fig. 1). Initially, no signs of respiratory distress were observed. Oxygen saturation was adequate and there were no signs of hemodynamic instability.

Clinical investigation revealed multiple extensive soft tissue injuries: in the occipital region, subnasally extending from cheek to cheek and through the tongue, which was almost completely avulsed. There was profound arterial 
Table 1 Indications for tracheotomy in maxillofacial trauma patients

Acute airway obstruction

Prolonged mechanical ventilation

Multiple facial fractures combined with basilar skull fractures

Complete destruction of the nasal anatomy combined with

multiple facial injuries

Patients admitted with preexisting cricothyrotomy

bleeding from the lacerations and oral cavity. There was gross displacement of the right globe with telecanthus and abnormal mobility of the entire viscerocranium. There were no obvious signs of injury to the neurocranium.

Because of a significant risk of loss of airway patency, the risk of vomiting with subsequent aspiration, and the need for further radiological evaluation and surgical therapy, a secure airway was necessary. Extreme difficulties were to be expected with facemask ventilation, laryngoscopy, and endotracheal intubation. Therefore, an invasive airway access was chosen. At that moment, the patient was still able to maintain a patent airway without signs of respiratory distress. It was decided to perform a percutaneous wireguided dilation cricothyrotomy (Melker Emergency Cricothyrotomy; Cook Critical Care, Bloomington, IN, USA) in local anesthesia with the patient still in a prone position, to be followed by a surgical tracheotomy. After the application of local anesthesia; needle puncture; placement of the guide wire; dilatation; and, eventually, placement of the tube, the patient went into an acute respiratory failure. Physical diagnostic examination revealed that the tube was not inside the trachea as no return of carbon dioxide could be monitored and no pulmonary ventilation could be auscultated. An emergency surgical tracheotomy was then performed. This

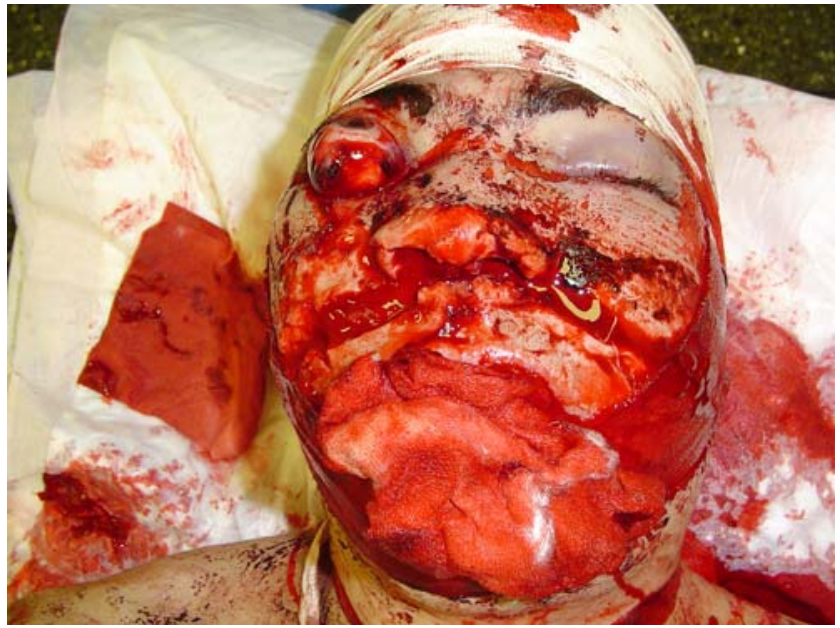

Fig. 1 Clinical aspect at presentation at the emergency ward procedure was uneventful and a cuffed trachea cannula was inserted. The patient was then sedated. Computed tomography of the thorax revealed air in the anterior upper mediastinum that was most likely due to false passage of the cricothyrotomy tube (Fig. 2). Fortunately, this potentially life-threatening event was without serious consequences as no signs of neurological deficits or infection of the mediastinum became present. Computerized tomography scanning of the head and neck revealed extensive midfacial and mandibular fractures (Fig. 3). The patient was operated upon and transferred to the intensive care unit where he stayed for 4 days before returning to the ward. The patient was decanulated successfully on the 16th postoperative day.

\section{Discussion}

Shortly after Seldinger (1953) described his technique of percutaneous guide wire needle placement in arteriography, the technique of percutaneous tracheotomy emerged [17]. Shelden et al. introduced a tracheotomy technique that consisted of a slotted needle guiding a trocar into the trachea [18]. Over the years, several modifications of the percutaneous technique evolved, and nowadays, there are numerous convenient PCT kits on the market based on different methods, utilizing different dilatators, guide wires, and points of entry into the trachea $[2,12,16,20]$.

PCT has been popularized as a safe and effective alternative to the conventional open tracheotomy technique. It is widely used in the intensive care unit setting, where it is now outnumbering the performance of COT [8]. In our institution, extensive experience has been gained with both the Ciaglia Blue Rhino tracheotomy and the guide wire dilating forceps technique, showing a low major complication rate $[6,7,9]$.

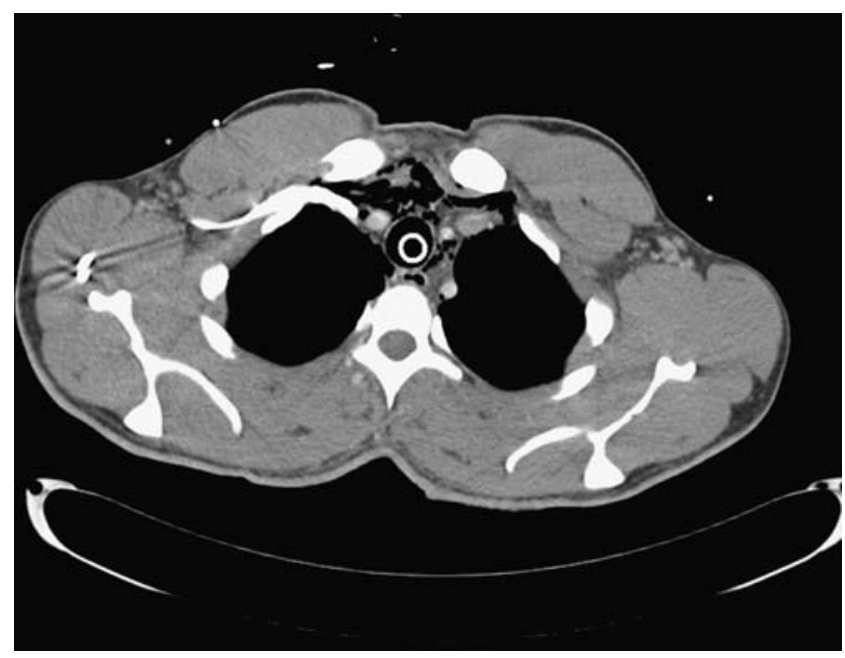

Fig. 2 Computerized tomography scan of the thorax with signs of mediastinal emphysema 


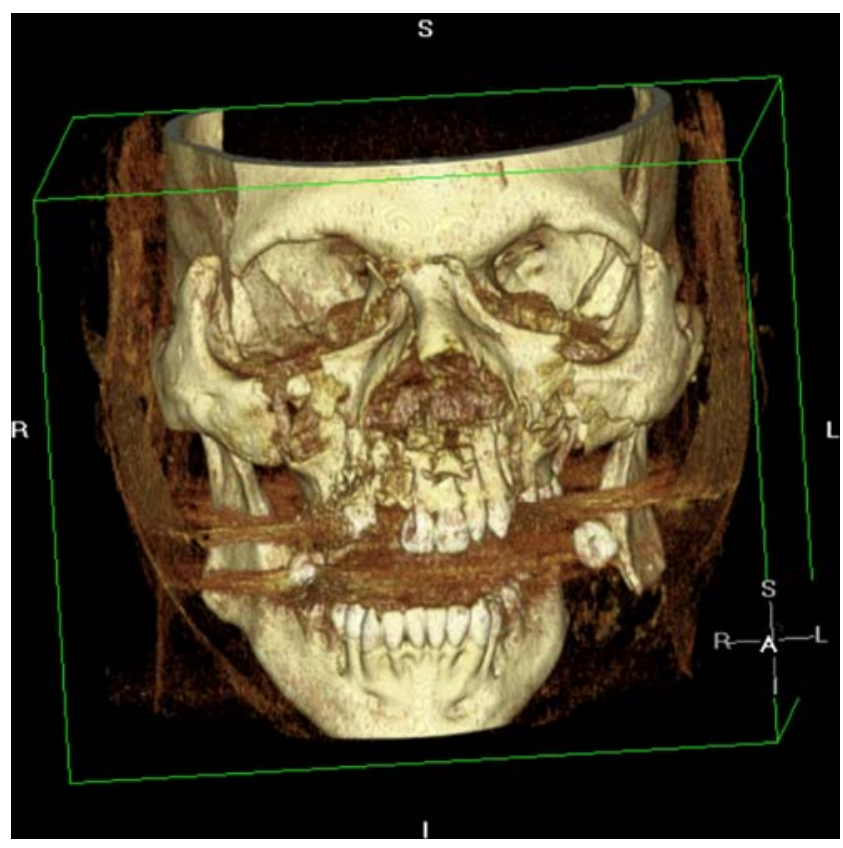

Fig. 3 Three-dimensional reconstruction of the maxillofacial skeleton showing gross destruction and displacement of the osseous structures

Various authors compare the open and percutaneous methods in terms of complication rates, cost-effectiveness, and safety. The potential for serious complications following PCT due to incorrect needle placement or posterior tracheal wall injury decreases with endoscopic guidance.

\section{Comparison of elective COT with PCT}

In a meta-analysis, Dulguerov et al. compared the peri- and postoperative complications of PCT and COT and concluded that the percutaneous variant is associated with more peroperative complications, especially concerning cardiorespiratory arrests and peroperative deaths. In addition, false passage of the tube was more frequent with PCT [5]. A double-blind randomized trial by the same authors led to the conclusion that both methods are associated with a low rate of serious and intermediate complications but that there were more minor peroperative complications with PCT and more minor long-term complications with COT [13]. In a prospective randomized trial, Massick et al. found no differences in peroperative complication rates between PCT and COT, but a significantly higher postoperative complication rate with PCT was found [14]. Goldenberg et al. found similar and low complication rates for COT and PCT [11]. In a review paper, McWhorter concludes that COT can be performed with equal or better outcome than PCT [15].

The differences between these studies can be attributed to study design, definitions, sample size, variance in patient population, and application of different surgical and percutaneous techniques. In these studies, PCT and COT were mainly performed as elective procedures in already intubated patients. Overall, it can be stated that both COT and PCT can be performed with low and acceptable complication rates when performed electively, assuming that experienced operators perform these procedures.

\section{PCT in an emergency situation}

In an emergency situation where rapid airway access is needed because of acute or impending airway obstruction and where nasal or oral endotracheal intubation is not possible or fails, a cricothyrotomy provides the most rapid means for securing the airway (although this is not regarded as a definitive airway). The use of PCT in an emergency situation is less clear and it has been stated that there is no role for such techniques in these situations $[2,15]$. The literature regarding this topic is sparse, although some reports assess this topic $[1,3,4]$.

Ben-nun et al. describe 10 patients with multiple blunt head and neck injuries or severe head and neck burns in which airway control was necessary. PCT was applied after oral endotracheal intubation failed. All patients were in the supine position, and on average, it took $5.5 \mathrm{~min}$ to perform this procedure. Experienced surgeons performed all procedures. No conversion to an open tracheotomy was necessary [1]. A case series by Clarke et al. describes PCT in four patients. Indications were inability to intubate, epiglottitis, Ludwig's angina, and swelling of the tongue. Two of these patients were in a semiprone position, and in all four cases, PCT was performed rapidly and safely [3]. The performing surgeon had an experience of over 200 elective PDT. In another two cases with airway obstruction and respiratory failure PCT, was performed successfully and fast [4]. Fikkers et al. compare the wire-guided Minitrach II to the catheter over needle cricothyrotomy (Quicktrach) in a pig model. A cricothyrotomy with the Quicktrach could be performed faster (48 vs $150 \mathrm{~s}$ ), easier, with a higher success rate, and with fewer complications than the wire-guided technique [10].

No clinical randomized trials are known that compare PCT with COT in a situation where urgent airway control is necessary. In the case descriptions that are available, almost all patients were in a supine position. A limited ability to extend the cervical spine is considered a contraindication for PCT [11]. Utilizing the wire-guided percutaneous technique in the patient described most probably led to a false route of the tube and loss of airway patency. In addition, computed tomography revealed air in the upper mediastinum, which theoretically increases the probability of mediastinitis. The prone position of this patient, as well as the experience of the performing surgeon, may have contributed to this complication. 


\section{Conclusion}

PCT has proven its merits in an elective nonemergency situation in the supine patient. No large series are known referring to the safety and feasibility of PCT in an emergency situation. There are, nonetheless, several reports that discuss the potential of this technique in such a situation, again in patients in the supine position and always performed by surgeons with a large experience in PCT. Limited ability to extend the cervical spine is considered a contraindication for PCT. In the patient described, the attempt to perform a percutaneous wireguided dilation cricothyrotomy in a sitting position in all probability led to acute deterioration of airway patency and mediastinal emphysema. It is therefore concluded that percutaneous cricothyrotomy or tracheotomy in prone patients with severe maxillofacial trauma is not an appropriate first-choice method and that either a catheter over needle cricothyrotomy or open surgical tracheotomy under local anesthesia should be performed when the situation necessitates acute airway control.

Open Access This article is distributed under the terms of the Creative Commons Attribution Noncommercial License which permits any noncommercial use, distribution, and reproduction in any medium, provided the original author(s) and source are credited.

\section{References}

1. Ben-nun A, Altman E, Best LA (2004) Emergency percutaneous tracheostomy in trauma patients: an early experience. Ann Thorac Surg 77:1045-1047

2. Ciagli P, Firsching R, Syniec C (1985) Elective percutaneous dilatational tracheostomy. A new simple bedside procedure; preliminary report. Chest 87:715-719

3. Clarke J, Jaffery A (2004) How we do it: emergency percutaneous tracheostomy: a case series. Clin Otolaryngol 29:558-561

4. Dob DP, McLure HA, Soni N (1998) Failed intubation and emergency percutaneous trachestomy. Anaesthesia 53:72-74
5. Dulguerov P, Gysin C, Perneger T, Chevrolet JC (1999) Percutaneous or surgical tracheostomy: a meta-analysis. Crit Care Med 27:1617-1625

6. Fikkers BG, Briede IS, Verwiel JM, van den Hoogen FJ (2002) Percutaneous tracheostomy with the blue rhino technique: presentation of 100 consecutive patients. Anaesthesia 57:1094-1097

7. Fikkers BG, van Heerbeek N, Krabbe PF, Marres HA, van den Hoogen FJ (2002) Percutaneous tracheostomy with the guide wire dilating forceps technique: presentation of 171 consecutive patients. Head Neck 24:625-631

8. Fikkers BG, Fransen GAJ, van der Hoeven JG, Briedé IS, van den Hoogen FJ (2003) Tracheostomy for long-term ventilated patients: a postal survey of ICU practice in The Netherlands. Intensive Care Med 29:1390-1393

9. Fikkers BG, Staatsen M, Lardenoije SGGF, van den Hoogen FJA, van der Hoeven JG (2004) Comparison of two percutaneous tracheostomy techniques, guide wire dilating forceps and ciaglia blue rhino: a sequential cohort study. Crit Care 8:R299-R305

10. Fikkers $B G$, van Vugt $S$, van der Hoeven JG, van den Hoogen FJA, Marres HAM (2004) Emergency cricothyrotomy: a randomised crossover trial comparing the wire-guided and catheterover-needle techniques. Anaesthesia 59:1008-1011

11. Goldenberg D, Golz A, Huri A, Netzer A, Joachims H, Bar-Lavie Y (2003) Percutaneous dilation tracheotomy versus surgical tracheotomy: our experience. Otolaryngol Head Neck Surg 128:358-363

12. Griggs WM, Worthley LIG, Gilligan JE, Thomas PD, Myburg JA (1990) A simple percutaneous tracheostomy technique. Surg Gynecol Obstet 170:543-545

13. Gysin C, Dulguerov P, Guyot JP, Perneger TV, Abajo B, Chevrolet JC (1999) Percutaneous versus surgical tracheostomy: a double-blind randomized trial. Ann Surg 230:708-714

14. Massick DD, Yao S, Powell DM, Griesen D, Hobgood T, Allen JN, Schuller DE (2001) Bedside tracheostomy in the intensive care unit: a prospective randomized trial comparing open surgical tracheostomy with endoscopically guided percutaneous dilational tracheotomy. Laryngoscope 111:494-500

15. McWhorter AJ (2003) Tracheotomy: timing and techniques. Curr Opin Otolaryngol Head Neck Surg 11:473-479

16. Schachner A, Ovil Y, Sidi J, Rogev M, Heilbronn Y, Levy M (1989) Percutaneous tracheostomy: a new method. Crit Care Med 17:1052-1056

17. Seldinger SI (1953) Catheter replacement of the needle in percutaneous arteriography. Acta Radiol 39:368-376

18. Shelden CH, Pudenz RH, Freshwater DB, Crue BL (1955) A new method for tracheotomy. J Neurosurg 12:428-431

19. Taicher S, Givol N, Peleg M, Ardekian L (1996) Changing indications for tracheostomy in maxillofacial trauma. J Oral Maxillofac Surg 54:292-295

20. Toy FJ, Weinstein JD (1969) A percutaneous tracheostomy device. Surgery 65:384-389 